\title{
Entre rhétorique et dramaturgie : la lecture de Térence par Mélanchthon
}

\section{Christiane Deloince-Louette}

\section{(2) OpenEdition}

1 Journals

Édition électronique

URL : http://journals.openedition.org/rhetorique/564

DOI : $10.4000 /$ rhetorique.564

ISSN : 2270-6909

Éditeur

UGA Éditions/Université Grenoble Alpes

Édition imprimée

ISBN : 978-2-37747-029-7

\section{Référence électronique}

Christiane Deloince-Louette, « Entre rhétorique et dramaturgie : la lecture de Térence par Mélanchthon », Exercices de rhétorique [En ligne], 10 | 2017, mis en ligne le 26 décembre 2017, consulté le 02 octobre 2020. URL : http://journals.openedition.org/rhetorique/564 ; DOI : https://doi.org/10.4000/ rhetorique.564

Ce document a été généré automatiquement le 2 octobre 2020.

\section{(c) () (ㅇ)}

Les contenus de la revue Exercices de rhétorique sont mis à disposition selon les termes de la Licence Creative Commons Attribution - Pas d'Utilisation Commerciale - Partage dans les Mêmes Conditions 4.0 International. 


\title{
Entre rhétorique et dramaturgie : la lecture de Térence par Mélanchthon
}

\author{
Christiane Deloince-Louette
}

1 L'intérêt du praeceptor Germaniae pour Térence s'est manifesté très tôt, et d'emblée dans un cadre scolaire. C'est à Tübingen que Mélanchthon a étudié les comédies du poète latin dans le cadre de ses cours sur l'Antiquité païenne et c'est dans cette ville que paraît sa première édition des six comédies en 1516 où il rétablit le texte dans ses vers ${ }^{1}$. Son édition commentée, qui date de 1528, montre l'approfondissement d'un projet pédagogique. Préfaces, arguments des comédies, notes marginales, tout un riche paratexte complété dans des éditions ultérieures, dit et redit cette orientation fondamentale. Écrivant à la fois pour les maîtres et pour les jeunes garçons, le professeur insiste sur la triple formation dont Térence est l'instrument : maîtrise de la langue (latine), formation des mœurs et amour des textes antiques dont le commentaire révèle la beauté ${ }^{2}$. Comme la tragédie, ou la comédie politique d'Aristophane, la comédie de Térence, qui peint les mœurs humaines, est un instrument utile pour former des futurs citoyens ${ }^{3}$.

Dans ce contexte, la mise en forme de l'édition "scolaire» a une importance non négligeable. Les arguments et notes marginales qui accompagnent les comédies de Térence définissent une "forme de l'intention " pour reprendre un titre célèbre de Michael Baxandall, tout autant qu'une forme de l'attention, si l'on me permet ce jeu facile. Car l'important est bien d'intéresser l'élève à Térence et, pour ce faire, d'exposer de la manière la plus claire et convaincante possible les grandes lignes de l'organisation d'une comédie. Mélanchthon applique aux textes de Térence une méthode qu'il pratiquera aussi pour l'épopée de Virgile ou pour les épîtres de saint Paul ${ }^{4}$. Mais ce faisant, il adapte et réoriente le commentaire de Donat, peut-être sous l'influence d'Aristote dont il cite la Poétique dans ses textes préfaciels. Nous suivrons donc ici, comme ses élèves le faisaient, l'itinéraire tracé par le pédagogue - les arguments des comédies, puis les notes marginales - pour tenter de saisir, non seulement un projet pédagogique mais une lecture rhétorique et critique, voire une poétique implicite de la comédie. 


\section{L'argument : au fondement de la pratique pédagogique}

3 Comme Donat, Mélanchthon fait précéder le texte de chacune des comédies de Térence d'un argumentum qui rend compte de la pièce. Qu'est-ce à dire? Argumentum est, dès l'Antiquité et comme son homologue français, un mot polysémique : il relève d'une part des pratiques de la persuasion, d'autre part, des formes de la narration. Dans son Institution oratoire, Quintilien note qu'il ne désigne pas seulement une preuve ou un motif de persuasion, mais aussi « une fable ou une fiction accommodée au théâtre ", voire " toute matière dont on fait choix pour écrire ${ }^{5}$ ». Cicéron, définissant la narratio dans son De l'Invention, distinguait plus précisément l'argumentum de la fabula et de l' historia. L'histoire rapporte des faits qui ont eu lieu dans le passé (« historia est gesta res, ab aetatis nostrae memoria remota »), la fable est un récit qui ne contient ni faits vrais ni faits vraisemblables ("fabula est in qua nec uerae nec ueri similes res continentur ») et l'argument est un récit fictif mais qui aurait pu arriver («argumentum est ficta res quae tamen fieri potuit $\left.{ }^{6} »\right)$. Et Cicéron de citer Térence, d'abord le récit de Simon au début de L'Andrienne pour illustrer l'argumentum, puis un discours rapporté prononcé par Micion au début des Adelphes pour illustrer «la narration qui concerne les personnes" (« narratio quae uersatur in personis »), c'est-à-dire le récit qui donne à voir « le langage et le caractère des personnages » (« personarum sermones et animi »).

4 L'usage éditorial de l'argumentum vise à rendre compte, de manière préliminaire, du sujet de la comédie ${ }^{7}$. En cela, il est bien un récit. Mais la vocation critique et pédagogique qu'il possède au $\mathrm{XVI}^{\mathrm{e}}$ siècle en fait plutôt une narratio au sens rhétorique du terme, c'est-à-dire un exposé des faits orienté par le désir de persuader. En témoigne la longue préface de Pierre Davantès (Antesignanus) à son édition de Térence de 1560 qui définit ainsi les argumenta :

Argumenta enim non solum Comoediarum, Actuum, et singularum Scenarum occasionem, et œconomiam, orationisque dispositionem ac distributionem, necnon partium fabulae ordinem et distinctionem planissime demonstrant, atque rerum summam complectuntur; sed etiam quid ex his, quod ad formandos hominum mores faciat, depromere debeant studiosi adolescentes ob oculos apertissime proponunt.

Les arguments en effet ne montrent pas seulement de façon très claire l'occasion (occasio) des Comédies, des Actes et des Scènes prises une à une, la conduite générale de la pièce (œconomia) et la disposition et distribution du dialogue, ils ne montrent pas seulement l'ordre et la distinction des parties de la pièce, et ne se contentent pas d'embrasser l'ensemble des matières; mais leur propos est aussi de mettre au grand jour et devant les yeux ce que les jeunes gens doivent en tirer, qui puisse contribuer à la formation des mœurs humaines ${ }^{8}$.

L'argument rend compte à la fois d'une dramaturgie et d'une éthique : il donne à voir l'organisation d'ensemble de la pièce (son économie, dira le XVII siècle) et en souligne l'utilité pour les mœurs. Mais, donnant à voir l'organisation d'ensemble, il est en même temps synthèse et analyse. Synthèse parce qu'il résume la matière (rerum summa); analyse, parce qu'il rend compte de chaque partie de la comédie et de la distribution de la parole. Et l'on peut penser que la mise en valeur de l'économie de la pièce sert la leçon éthique qu'il faut en tirer.

5 Aussi le but pédagogique de l'argument est-il doublement valorisé, et même triplement si l'on considère l'exercice dont il fait l'objet chez Mélanchthon. À la fin de sa préface à 
L'Andrienne, ce dernier précise : «Il faut exiger des enfants eux-mêmes qu'ils rédigent les arguments des pièces avec leurs propres forces, qu'ils recomposent un certain nombre de passages en les développant, et les remodèlent comme de la cire. Cette pratique, outre qu'elle favorise une connaissance plus intime du Poète, nourrit aussi l'aisance dans le discours ${ }^{9}$ ». Le maître doit les guider en ce sens :

In recensendis argumentis fabularum, consilium Poetae summa cura exponendum est. Quarum personarum descriptiones in primis admirationem mereantur, in quibus locis plurimum commoretur Poeta, ubi plurimum nervorum, plurimum eloquentiae ostentet, quos locos ita commendent pueris, ut familiariter ament, et tanquam digitos suos norint.

Lorsqu'on passe en revue les sujets (argumenta) des pièces, il faut exposer avec le plus grand soin le dessein du Poète et indiquer quels sont les portraits de personnages qui méritent en premier l'admiration, quels sont les passages sur lesquels le Poète s'est le plus attardé, là où il montre le plus de force, le plus d'éloquence, quels passages seront recommandés aux enfants pour qu'ils les apprécient intimement et les connaissent sur le bout des doigts ${ }^{10}$.

On retrouve le double aspect de synthèse (consilium Poetae) et d'analyse (personarum descriptiones, loci, etc.) propre à l'argument. Pour en rendre compte, le choix de la forme $\mathrm{du}$ récit est un élément déterminant. Notre pratique moderne n'y voit aucune difficulté: nous résumons ordinairement l'intrigue en respectant l'ordre des événements tels qu'ils sont présentés dans la fable. Or, si l'on regarde de près les arguments de Donat et ceux de Mélanchthon, on constate que ce choix ne va pas de soi.

\subsection{La forme d'un récit}

6 En effet, Donat recontextualise la comédie quand Mélanchthon suit le déroulement de l'intrigue. L'opposition relève de la poétique du récit que l'on trouve chez Horace : au récit $a b$ ovo, qui veut tout embrasser depuis l'origine, Horace préfère celui «qui emporte l'auditeur au milieu des faits comme s'ils étaient connus » (« in medias res / Non secus ac notas auditorem rapit $\left.{ }^{11} »\right)$. Quintilien juge de même que l'orateur doit décider, à propos de l'exposition des faits, «s'il faut qu'elle commence par l'origine des choses, ou par le milieu à la manière d'Homère, ou par la fin ${ }^{12}$ ». Voici, par exemple, la partie centrale de l'argument de Donat pour L'Andrienne qui rend compte des événements racontés dans la comédie :

Chremes Atticus, pater Pasibulae et Philumenae, cum ex his duabus Pasibulam perdidisse se falso crederet relictam Athenis nec uisam postea multo tempore, tamquam unicam sibi putabat Philumenam. quam Charinus adulescens Atheniensis et amabat unice et petebat uxorem, sed pater eam Pamphilo cuidam, Simonis filio, ultro desponderat. qui Pamphilus contra sororem Chrysidis ac peregrinam tum creditam, Pasibulam supra dictam, Chremetis alteram filiam ac sub nomine Glycerii latentem, sic amauit, ut ex ea filium suscepisset inscio patre. qua re intellecta commotus Simo, Pamphili pater, dum per falsas nuptias temptat animum Pamphili, multis dolis a Dauo ipse deluditur seruo, periculumque Charini et Pamphili et totus error inenodabilis usque ad eum finem est ductus, dum Athenas ueniens Andrius quidam Crito rem aperiat et nodum fabulae soluat. per quem agnita Pasibula recipitur a parentibus et traditur Pamphilo amanti; item Philumena Charino despondetur et traditur exoptanti.

Un citoyen de l'Attique, Chrémès, père de Pasibule et Philumène, croyait à tort avoir perdu l'une de ses deux filles, Pasibule, qu'il avait laissée à Athènes et n'avait pas vue ensuite pendant un long moment. Il considérait donc Philumène comme sa fille unique. De cette Philumène s'était épris un jeune Athénien, Charinus; il n'aimait qu'elle et voulait l'épouser, mais son père l'avait de son côté promise à un dénommé Pamphile, fils de Simon. Ce Pamphile, quant à lui, avait conçu un tel amour pour la sœur de Chrysis, que l'on pensait étrangère, mais qui était en réalité 
la Pasibule dont on a parlé, seconde fille de Chrémès, vivant sous le nom d'emprunt de Glycère, qu'il avait eu d'elle un fils à l'insu de son père. Comprenant cela, Simon est bouleversé et, tandis qu'il met à l'épreuve les sentiments de Pamphile en lui proposant un faux mariage, il se fait jouer lui-même par les ruses nombreuses de son esclave Dave; le péril que courent Charinus et Pamphile et la méprise ne se dénouent que dans la fin, quand un Andrien, Criton, venant à Athènes, révèle la vérité et résout le nœud de la pièce. Par son entremise Pasibule est reconnue, elle est rendue à ses parents et elle est accordée en mariage à son amant Pamphile; de même Philumène est promise à Charinus et elle lui est accordée en mariage selon son souhait ${ }^{13}$.

7 La première moitié $d u$ récit de Donat rassemble des faits nécessaires à la compréhension de la comédie mais extérieurs à elle. Nous ne saurons en effet qu'à la toute fin de la pièce que Glycère est Pasibule. Le sujet de la comédie est expédié en quelques mots : " un faux mariage », " des ruses nombreuses », l'arrivée de Criton qui "résout le nœud de la pièce » et permet la reconnaissance et la fin heureuse. Donat indiquera cependant dans un deuxième temps que le poète a adopté un type de composition circulaire qui fait commencer la comédie par les derniers éléments de l'histoire, type déjà adopté par Homère et Virgile ${ }^{14}$.

Lorqu'il publie en 1555 ses annotations aux comédies de Térence, Muret prend soin de rédiger à son tour un argumentum pour chaque pièce. Il prend le même parti que Donat, exposant d'abord la situation de Chrémès et de Phania, deux frères athéniens, puis le départ de Phania et son naufrage, l'arrivée de Chrysis et de Pasibule-Glycère à Athènes, etc. Il reconstruit donc le cadre complet de la comédie en brossant le portrait de chaque personnage important avant d'en venir à la ruse du faux mariage. Il résume alors minutieusement la pièce jusqu'à sa conclusion, en reprenant le modèle proposé par Donat qui recontextualise la pièce avant d'en donner l'intrigue ${ }^{15}$.

9 Tout autre est l'argument de Mélanchthon dont l'ordre est fondé sur la division en trois temps - protase, épitase et catastrophe - que décrit le De fabula d'Évanthius, un texte que l'âge classique attribue à Donat lui-même ${ }^{16}$. L'Andrienne est ainsi présentée :

Periculum PROTASIS [PROTHESIS en 1528]

Cum amaret Pamphilus Glycerium, eique fidem dedisset, ducturum se esse uxorem, Pater, qui amare filium compererat, consilium capit abstrahendi eius a Glycerio, simulat se uxorem daturum, poscitque ut ne filius id recuset. is cum et patrem uereretur, et Glycerium deserere parum pium iudicaret, pendet animi, nec habet, quo se uertat : ibi Dauus intelligens simulari rem a patre consulit herili filio, ut polliceatur patri, se in eius potestate futurum : periculi nihil esse, quandoquidem simulentur nuptiae. Filius obsequitur, quanquam aegre, pollicetur patri ducturum se quam uellet.

EPITASIS

Interea spargitur de Pamphili nuptiis rumor: resciscunt mulierculae, quibus cum Glycerio consuetudo erat. Et pater cum a filio quod uoluerat, extorsisset, non iam simulat rem, sed seriam fabulam orditur. Chremetem orat, ut gnatam det uxorem filio suo. Facile impetrat. Hic de Pamphilo actum fuit: maturare nuptias pater studet: Et filius pollicitus erat, se in patris potestate futurum esse. Quaeso, quomodo ex his turbis se explicet? quid faciat Pamphilus? Hic Davus, cujus imprudentia periculum contractum erat, cum dolis non successisset Chremetem deterret a nuptiis, fidem faciens, amari aliam a Pamphilo.

CATASTROPHÈ

At pater rem urget, et processisset, nisi casus, qui saepe plus valet, quam ratio, dubiis rebus opem attulisset. Venit quispiam Crito, qui docet Glycerium Chremetis filiam esse, gratum omnibus nuncium. Nuptiae ex sententia Pamphili conficiuntur. Vide ex quanto periculo, casu euaserit Pamphilus.

Le péril : EXPOSITION [PROTASE]

Alors que Pamphile aimait Glycère et lui avait promis de la prendre pour épouse, 
son Père, ayant appris que son fils était amoureux, forme le dessein de l'arracher à Glycère, feint qu'il va lui donner une épouse, et exige de son fils qu'il ne refuse pas la chose. Ce dernier, parce que tout ensemble il respecte son père et juge qu'il n'est guère pieux d'abandonner Glycère, est dans l'anxiété et ne sait où se tourner. C'est là que Dave, comprenant qu'il s'agit d'un faux-semblant préparé par le père, conseille au fils de promettre à son père qu'il s'en remettra à lui : il n'y a aucun péril puisque les noces sont feintes. Le fils cède, bien qu'à contre-cœur, et promet à son père qu'il épousera celle qu'il désire.

NEEUD [ÉPITASE]

Pendant ce temps, la rumeur du mariage de Pamphile se répand: les femmes qui sont dans l'intimité de Glycère viennent à le savoir. Et le père, comme il a arraché à son fils ce qu'il voulait, ne fait plus semblant mais commence à jouer sérieusement. Il prie Chrémès de donner sa fille pour femme à son propre fils. Il l'obtient facilement. Ici, c'en est fait de Pamphile : son père s'applique à hâter le mariage ; et le fils avait promis qu'il s'en remettrait à son père. Je vous le demande, comment va-t-il se sortir de ce trouble? Que va faire Pamphile? Ici Dave, dont l'imprudence était à l'origine $d u$ péril puisque sa ruse n'avait pas réussi, détourne Chrémès $d u$ mariage en lui faisant croire que Pamphile en aime une autre.

DÉNOUEMENT [CATASTROPHE]

Cependant le père presse les choses et aurait réussi si le hasard, qui souvent fait mieux que la raison, n'avait apporté son aide dans cette situation incertaine. Arrive un certain Criton qui leur apprend que Glycère est la fille de Chrémès, messager agréable à tous. Le mariage se fait comme le voulait Pamphile. Vois de quel grand péril Pamphile a été sauvé par le hasard !

Il ne s'agit plus de récit chronologique mais d'une présentation dramatique de la comédie. Plusieurs éléments y contribuent: la fidélité à l'ordre des faits tels qu'ils apparaissent dans la pièce ; la mise en valeur de la cohérence de cette dernière grâce à la notion de péril (periculum) empruntée à Donat; le recours à une narration adressée qui, en mimant la tension croissante par des interrogations, souligne les moments dramatiques où le suspense est à son comble, en particulier dans l'épitase. Mélanchthon privilégie l'ordre voulu par le poète, c'est-à-dire un ordre "artificiel », conçu pour agir sur le spectateur et le maintenir en haleine. L'argumentum est chez lui rhétorique au sens où, non seulement, il reproduit la logique de la pièce mais aussi valorise l'effet produit.

\subsection{Une lecture rhétorique?}

11 Revenons sur la tripartition antique (veteri more) de la comédie, et sur les termes que Mélanchthon utilise pour mieux en montrer les ressorts. La préface de L'Andrienne, très éclairante à cet égard, commence par distinguer trois notions clés : periculum, le péril qui menace un personnage et le plonge dans un océan de troubles; consilium, la délibération, en est la conséquence car « Il n'y a que dans les situations critiques que la délibération est nécessaire " ("Nusquam enim consilio locus est, nisi in dubiis rebus»); enfin status causae ou stasis fabulae, l'état de la cause, ici conjectural : le mariage aura-til lieu? Toute la pièce, dit Mélanchthon, est semblable à un discours de genre délibératif ${ }^{17}$. Ce n'est cependant pas le cas systématiquement. Que l'on considère en effet la comédie de L'Eunuque, qui a pour personnage principal le soldat fanfaron Thrason : «Ici, il y a davantage de discours de reproche et la pièce est plus proche du genre accusatoire ou judiciaire » (« Hic plus est expostulationum, et accusatorio seu iudiciali generi similior est »). Dans un cas, periculum, consilium, genre délibératif; dans l'autre, periculum, expostulatio, genre judiciaire. La notion dramaturgique de " péril » se déploie 
selon des catégories rhétoriques hiérarchisées (types de discours, genre) conformément au projet pédagogique qui veut que la lecture de Térence prépare aussi à l'apprentissage de l'éloquence. Si, pour Marc Fumaroli - et pour Corneille - « Le vœu profond de la rhétorique, c'est de s'accomplir en dramaturgie ${ }^{18}$ ", Mélanchthon propose une lecture inverse : le vœu de la dramaturgie, c'est de s'accomplir en discours.

12 Mais la dramaturgie revient en force grâce au recours à la terminologie grecque empruntée à Évanthius et reprise par Donat. Mélanchthon ne perd donc pas de vue l'organisation générale propre à la comédie. Les trois termes protasis, epitasis, catastrophè, donnés en caractères grecs dans la marge, sont accompagnés de leurs homologues latins : propositio, intentio, conversio, qui ne seront pourtant pas repris par la suite dans les notes marginales du texte. Si propositio pourrait avoir un sens rhétorique (à savoir l'essentiel du propos, le cœur du sujet - summa rei, dit Mélanchthon dans ses Elementa rhetorices ${ }^{19}$ ) et désigner en même temps l'exposition, les deux autres termes s'éloignent du modèle qu'offre le discours. Intentio et conversio, le nœud et le dénouement ou, plus exactement, la tension et le renversement, sont en effet deux parties de la comédie données comme inséparables. L'intentio désigne la montée en puissance du periculum et conversio le retour au calme qui fait disparaitre tout péril ${ }^{20}$. L'opposition entre les deux est celle qui existe entre le désordre et le retour à l'ordre, entre une tension - un suspense - que l'on conduit à son point culminant et l'apaisement qui suit le renversement de la situation ${ }^{21}$. Dans L'Andrienne, la propositio consiste dans le trouble de Pamphile qui veut tout à la fois conserver Glycère qu'il aime et obéir à son père qui veut lui faire épouser la fille de Chrémès. L'intentio tient aux agissements de Dave : croyant qu'il s'agissait d'un faux mariage, il a poussé Pamphile à dire oui à son père. Mais Simon veut rendre le mariage réel : comment s'en sortir ? La conversio est permise par l'arrivée de Criton qui révèle que Glycère n'est autre que la fille perdue de Chrémès. Le choix du couple intentio/conversio ou epitasis/catastrophè est donc nettement un choix dramaturgique qui met l'accent sur la construction antithétique de la comédie, à la fois en termes de structure (on noue/on dénoue) et en termes d'effet produit sur le spectateur (tension/apaisement ${ }^{22}$ ).

Tout cela a le mérite de la clarté, lorsqu'il s'agit de faire comprendre aux élèves quels sont les ressorts de la comédie. On a remarqué que nulle part dans ces textes préfaciels, Mélanchthon n'évoque la question des actes (alors même qu'Évanthius-Donat dans le De comoedia tente d'associer les étapes abstraites que sont la protase, l'épitase et la catastrophe avec les divisions concrètes que constituent les actes) et cette absence semble pouvoir se justifier encore une fois par le but pédagogique qu'il poursuit : saisir le mouvement général de la comédie comme on saisit le mouvement général d'un discours, saisir une dynamique quand le découpage en actes pourrait sembler figer artificiellement l'action.

Tous les arguments rédigés par Mélanchthon ont recours à cette présentation en trois temps. Mais les hésitations que révèlent ceux de L'Eunuque et l'Heautontimoroumenos mettent en valeur l'originalité de ces deux comédies.

\subsection{Deux cas particuliers : L'Eunuque et l'Heautontimoroumenos}

15 L'argument de L'Eunuque permet en effet de voir la complexité d'une pièce qui comporte deux épitases et donc deux dénouements. 
Superiori fabula proposuit modesti adolescentis exemplum. Is de officio suo honeste consultat. Hic nulla sunt honesta exempla, nullum honestum consilium. Nam meretricii amores describuntur, ut in hoc exemplo cernant iuuenes tanquam in speculo, amentiam huiusmodi amatorum. Est autem duplex propemodum fabula, propterea geminum argumentum recenseo.

Primas Thraso in hac fabula tenet. Et cum pollicita esset Thais, facturam se sui copiam dies aliquot, fallitur is tamen: Eaque protasis fabulae est.

Cum Thrasone confertur Phaedria : nisi enim riualem haberet Thraso, nihil periclitaretur in amore.

Epitasis fabulae est, cum minatur ille se oppugnaturum in aedeis Thaidis.

Catastrophe ridicula est. Nam Thraso iam fractus precibus impetrat, ut aliqua saltem in parte apud Thaidem haereat.

Porro quia haec fabula uaria est, multumque habet turbarum, casu exoritur alius tumultus, uitiatur uirgo, quam Thaidi miles donarat. Sed est laeta eius quoque periculi catastrophe. nam uxor datur haec adolescenti, quam compresserat.

$V t$ Thrasonem ridiculum amatorem fingeret, addit Gnathonem, qui eius mores mirifice depingit, ut ardelionem agnoscas. [...]

La pièce précédente a mis en avant l'exemple d'un jeune homme modeste. Ce dernier délibère avec honnêteté sur son devoir. Mais ici, nul exemple honorable, nul dessein honnête. Car ce sont des amours vénales qui sont décrites afin que, dans cet exemple, les jeunes gens regardent, comme en un miroir, la folie de cette sorte d'amants. Il y a presque une double pièce, par conséquent j'en rapporte les deux arguments conjoints.

Thrason joue le premier rôle dans cette pièce. Et alors que Thaïs lui avait promis de lui ouvrir sa porte durant quelques jours, il est cependant trompé : c'est l'exposition [protase] de la pièce.

À Thrason est confronté Phédria. Car si Thrason n'avait pas de rival, rien ne mettrait en péril son amour.

Le nœud [épitase] de la pièce a lieu lorsqu'il menace de donner l'assaut à la demeure de Thaïs.

Le dénouement [catastrophe] est drôle. Car Thrason, réduit à néant, obtient par ses prières de tenir au moins quelque rôle auprès de Thaïs.

En outre, parce que cette pièce est variée et contient beaucoup d'éléments de trouble, un autre désordre naît par hasard : la jeune fille que le soldat avait donnée à Thaïs est violée. Mais le dénouement de ce péril est aussi joyeux. Car elle est donnée pour épouse au jeune homme qui lui avait fait violence.

Pour représenter Thrason en amant ridicule, il a ajouté Gnathon qui dépeint ses manières avec admiration, afin que tu connaisses le type du flatteur.

16 Une même comédie peut donc comporter deux intrigues, deux épitases et deux catastrophes. L'intrigue principale concerne ici le personnage de Thrason, soldat fanfaron, qui fait effort pour conquérir Thaïs malgré son rival Phédria. La deuxième intrigue concerne la jeune fille violée. Dans les deux cas, le trouble atteint son point culminant (l'assaut de la maison de Thaïs, la découverte du viol) avant de trouver sa résolution (l'arrangement conclu avec Thaïs, le mariage de la jeune fille). La composition de l'argument, plus lâche ici, témoigne d'une certaine gêne du commentateur, mais c'est bien la structure en deux temps majeurs - épitase/ catastrophe - qui permet de mettre en lumière la double intrigue. Remarquons qu'il n'y a rien de tel chez Donat pour qui prime l'unité de l'ensemble :

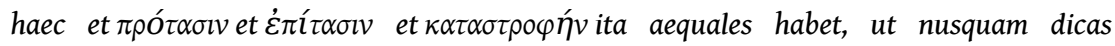
longitudine operis Terentium delassatum dormitasse. Actus sane implicatiores sunt in ea et qui non facile a parum doctis distingui possint, ideo quia tenendi spectatoris causa uult poeta noster omnes quinque actus uelut unum fieri, ne respiret quodammodo atque, distincta alicubi continuatione succedentium rerum, ante aulaea sublata fastidiosus spectator exsurgat. 
La pièce montre dans la protase, l'épitase et la catastrophe une si grande égalité que l'on pourrait dire que nulle part Térence, malgré la longueur de l'œuvre, n'a éprouvé de fatigue ou s'est endormi. Les actes y sont particulièrement imbriqués et difficiles à séparer si l'on n'est pas tant soit peu spécialiste, parce que, pour tenir en haleine le spectateur, notre poète veut que les cinq actes n'en fassent pour ainsi dire qu'un, afin qu'il n'ait pas le temps de respirer et que, devant un enchaînement d'événements qui se succèdent avec de rares pauses ici ou là, le spectateur fatigué ne quitte pas le théâtre avant le lever de rideau ${ }^{23}$.

\section{L'argument de l'Heautontimoroumenos montre une autre forme de complexité.}

Depingit autem parentum uitia. Nam Menedemus liberaliter amantem Cliniam iusto inclementius tractat adolescentem modestum et frugi. Quem postquam domo eiecit uictum iurgiis et contumeliis, multo impotentius desiderat.

Adeo multifariam hic peccat. Huic seni opponit Chremetem monitorem, sed quem postea uideas plura quam Menedemum peccare. Primum is nunquam olfecit perdite amare filium, et compilari se a scorto sumptuoso. Post ubi resciscit, multo fert impotentius id mali, quam illa solebat Menedemus, ut agnoscas fere ita accidere, ut foris, non domi sapiamus.

EPITASIS

Porro quia fingebat poeta uelut oculis captum Chremetem, qui insignia filii uitia non cernat, adducit patri ob oculos filii amicam, idque hic periculi est, ne resciscat Chremes Bacchidem filii amicam esse. Huc omnia consilia, omnes dolos seruuli referemus. Et a Chremete prius argentum emungitur Bacchidi numerandum, quam comperit filii rem agi. Fallitur autem cum fingitur Bacchis esse Cliniae.

In prima fabulae parte, ubi Menedemum confirmat Chremes, oratio est deliberativi, seu suasoriii generis, qua hic consolatur, erigit, monet animi aegrum vicinum.

In reliqua fabulae parte, quae periculum continet, res omnis translatione rhetorica agitur, quia quae ad Chremetis filium pertinent, omnia imputantur Menedemi gnato.

Elle dépeint les vices des pères. Car Ménédème traite Clinias de manière plus inclémente qu'il n'est juste, alors que son amour est noble et qu'il est un jeune homme mesuré et sage. Mais, après qu'il l'a chassé de la maison, vaincu par les reproches et les injures, il le regrette sans modération.

Il pèche donc en beaucoup d'endroits. À ce vieillard, le Poète oppose Chrémès le sermonneur ; mais c'est lui qu'on voit par la suite pécher plus que Ménédème. D'abord, il ne se rend jamais compte qu'il aime éperdument son fils, et qu'il est dépouillé par une courtisane qui mène grand train. Ensuite, lorsqu'il l'apprend, il supporte ce malheur de manière moins modérée encore que Ménédème ses maux précédents, pour que tu apprennes que parfois, nous savons ce qui se passe ailleurs mais non chez nous.

NCEUD [ÉPITASE]

En outre, parce que le Poète représentait Chrémès comme aveuglé, parce qu'il ne voit pas les vices insignes de son fils, il conduit au père, sous ses yeux, l'amie de son fils. Là est le péril : il faut empêcher que Chrémès apprenne que Bacchis est l'amie de son fils. C'est à cela que nous rapportons tous les projets, toutes les ruses du jeune esclave. Et de l'argent est soutiré à Chrémès pour être compté à Bacchis, avant qu'il ne découvre la situation de son fils. Mais il est trompé puisque Bacchis est présentée comme appartenant à Clinias.

Dans la première partie de la pièce, où Chrémès réconforte Ménédème, le discours relève du genre délibératif, ou persuasif : il console, raffermit, exhorte son voisin qui souffre en son cœur.

Dans le reste de la pièce, qui contient le péril, la matière dans son ensemble est traitée par transposition rhétorique, parce que tout ce qui concerne le fils de Chrémès est imputé au fils de Ménédème ${ }^{24}$.

18 L'argument se concentre d'abord sur les caractères des personnages, très précisément des pères: dans cette comédie stataria (avec peu d'action), l'intrigue est finalement secondaire par rapport à la dimension éthique qu'elle sert. Il y a cependant un periculum, qui concerne les fils. L'énormité de la ruse ici employée - faire croire que 
Bachis est l'amie de Clinias quand elle est celle de Clitiphon - ne fait que souligner l'aveuglement de Chrémès. L'argument reste très elliptique : le point culminant du péril - la découverte de la supercherie - est à peine évoqué, et la catastrophe - la réconciliation du père et $d u$ fils - n'est pas mentionnée. Au détriment d'une lecture dramatique de la pièce qui insisterait sur le péril qui menace Clitiphon, Mélanchthon privilégie la lecture éthique qui reconstruit la pièce autour de l'aveuglement de Chrémès. Le problème est bien le dessein du Poète, qui n'est pas toujours clair. Aussi les notes marginales sont-elles là pour le préciser.

\section{Les notes marginales : dégager la construction d'ensemble}

19 Les notes que Mélanchthon place dans les marges de son édition sont succinctes et peu nombreuses. Les unes ont pour but de repérer les étapes de la pièce telles qu'elles ont été résumées dans l'argument, les autres, exclusivement rhétoriques, précisent des types de discours, des figures, ou des émotions. Je m'attacherai ici exclusivement aux premières, en tant qu'elles retracent le dessein du Poète ou la conduite de la pièce.

\subsection{Séquences ou processus?}

Reprenons l'exemple de L'Andrienne, en suivant cette fois les indications inscrites en marge. La protase ne donne lieu à aucune note, peut-être à cause de la présence de Sosie, que Donat dans sa préface nomme justement personnage protatique. Cependant, l'argument de Mélanchthon donnait pour fin de la protase est la promesse de Pamphile à son père ("Filius obsequitur, quanquam aegre, pollicetur patri ducturum se quam vellet »), c'est-à-dire la scène 5 de l'acte II (v. 420). Mais on peut aussi considérer la protase comme une longue séquence qui ne s'achève qu'avec le début de l'épitase, que Mélanchthon note très précisément au début de la scène 3 de l'acte III : «Ex fictis nuptiis verae fient, idque initium est epitaseos periculi in fabula» («Les noces, de feintes, deviennent réelles, et c'est le commencement de l'épitase dans la pièce ${ }^{25} »$ ).

On entre alors dans une séquence de troubles. Au tout début de la scène suivante (III, 4), on trouve en marge : "Ad epitasin periculi pertinet quod eo res redit, ut Davi consilia obsint Pamphilo.» («Montée en croissance de la tension: les conseils de Dave portent préjudice à Pamphile»). Le péril se resserre autour de Pamphile, ce que montre encore la note en IV, 3 : «Audax consilium, quo res magis perturbata fuisset, nisi casu emersisset ex his malis Pamphilus» («Dessein hardi, qui aurait plus encore perturbé la situation si Pamphile ne s'était tiré de ces malheurs par un heureux hasard»). Dave cherchait en effet à sauver la situation en déposant le nouveau-né devant la porte de son maître. Le terme de casus annonce ici l'arrivée de Criton, donc la résolution des troubles. La fin de l'épitase est également notée, au tout début de la scène 1 de l'acte $V$ qui confronte Chrémès à Simon: "Ad finem epitaseos facit haec noua turba " (Ce nouveau trouble contribue à la fin de l'épitase »). Qu'est-ce que ce nouveau trouble ? Simon déclare à son ami que la présence d'un nouveau-né n'est qu'une machination de l'esclave. Dave a échoué une nouvelle fois à sauver son maître. La coupe est pleine, Pamphile est perdu, c'est le point culminant de la tension, donc la fin de l'épitase. 
Les séquences ne sont pourtant pas nettement distinctes car la catastrophe, implicitement évoquée dans la scène 3 de l'acte IV par le terme de casus, l'est explicitement dans la scène 5 où est annoncée l'arrivée de Criton : « Occasio catastrophes, nam senex casu interveniens, discutit periculum » («C'est l'occasion du dénouement, car l'arrivée fortuite du vieillard dissipe le péril »). La catastrophe proprement dite, c'est-àdire la révélation de Criton qui déclare que Glycère est citoyenne athénienne donc épousable, se produit dans la scène 4 de l'acte $\mathrm{V}$, avec l'arrivée effective du personnage («Catastrophe » en marge, au v. 906). Donat, lui, la signale dans la scène 2, lorsque Dave annonce à Chrémès l'arrivée de Criton. Donat semble repérer un processus long (la catastrophe s'étend de la scène 2 à la fin) quand Mélanchthon indique une séquence plus brève - les scènes 4 à 6 - qui a cependant été préparée - «occasio catastrophes » en IV, 5.

23 L'Eunuque et sa double intrigue offrent une annotation comparable. Pour plus de clarté, je la présenterai sous la forme d'un tableau.

\begin{tabular}{|c|c|c|}
\hline Mouvement de la pièce & Notes marginales & Traduction \\
\hline \multicolumn{3}{|l|}{ PROTASE } \\
\hline \multirow[t]{2}{*}{$\begin{array}{l}\text { Première } \\
\text { (Thrason) }\end{array}$} & $\begin{array}{l}\text { I, } 1 \text { : Opponit militi riualem } \\
\text { quem fingit aegre concedere } \\
\text { dies aliquot, dum a milite Thais } \\
\text { munus extorqueat. }\end{array}$ & $\begin{array}{l}\text { I, } 1 \text { : Il oppose au soldat un rival qu'il } \\
\text { représente lui concédant de mauvais } \\
\text { gré quelques jours, pendant que Thaïs } \\
\text { extorque un présent au soldat. }\end{array}$ \\
\hline & $\begin{array}{l}\text { I, 2: In hac scena, exponitur } \\
\text { occasio fabulae, ut Terentio } \\
\text { initio fabularum mos est. Simul } \\
\text { causa, cur miles sit placandus, } \\
\text { recensetur. }\end{array}$ & $\begin{array}{l}\text { I, 2: Dans cette scène, est exposée } \\
\text { l'occasion de la pièce, comme Térence } \\
\text { a l'habitude de le faire au début de ses } \\
\text { pièces. Est indiquée en même temps la } \\
\text { raison pour laquelle il faut apaiser le } \\
\text { soldat. }\end{array}$ \\
\hline $\begin{array}{l}\text { II, 3: Entrée en scène de } \\
\text { Chéréa } \\
\text { Parménon lui propose de } \\
\text { prendre la place de } \\
\text { l'eunuque. }\end{array}$ & $\begin{array}{l}\text { II, } 3 \text { : Occasio tamquam nouae } \\
\text { fabulae et noui motus in hac } \\
\text { comoedia. [...] } \\
\text { [v. } 364] \text { Ut mancipia haec] } \\
\text { Occasio consilii et noui motus. } \\
\text { [v. } 369] \text { Quid si] Consilium. }\end{array}$ & $\begin{array}{l}\text { II, 3: C'est comme l'occasion d'une } \\
\text { nouvelle pièce et d'une agitation } \\
\text { nouvelle dans cette comédie. } \\
\text { Pour emmener ces esclaves] Occasion du } \\
\text { dessein et de l'agitation nouvelle. } \\
\text { Et si c'était toi] Dessein. }\end{array}$ \\
\hline $\begin{array}{l}\text { Deuxième intrigue (la } \\
\text { jeune fille violée): Chéréa } \\
\text { raconte à Antiphon le viol } \\
\text { qu'il a commis sous le } \\
\text { déguisement d'eunuque. }\end{array}$ & $\begin{array}{l}\text { III, } 5 \text { : Narratio qua exponitur } \\
\text { protasis secundae partis in } \\
\text { fabula. }\end{array}$ & $\begin{array}{l}\text { III, } 5 \text { : Narration qui donne l'exposition } \\
\text { de la seconde intrigue de la pièce. }\end{array}$ \\
\hline
\end{tabular}




\begin{tabular}{|c|c|c|}
\hline $\begin{array}{l}\text { Gnathon conseille à } \\
\text { Thrason d'éveiller la } \\
\text { jalousie de Thaïs en faisant } \\
\text { l'éloge de Pamphila. }\end{array}$ & $\begin{array}{l}\text { III, } 1 \text { [v. 439]: Parasceuè } \\
\text { epitaseos in prima fabulae } \\
\text { parte. }\end{array}$ & $\begin{array}{l}\text { III, } 1 \text { : Préparation du nœud [mise en } \\
\text { place de la tension] en ce qui concerne } \\
\text { la première intrigue de la pièce. }\end{array}$ \\
\hline $\begin{array}{l}\text { Chrémès indique qu'il voit } \\
\text { Thrason qui arrive avec ses } \\
\text { troupes. }\end{array}$ & $\begin{array}{l}\text { IV, 6: } \text { Parasceù̀ eius motus, } \\
\text { quem miles excitabit } \\
\text { oppugnaturus aedes. }\end{array}$ & $\begin{array}{l}\text { IV, } 6 \text { : Préparation de l'agitation que le } \\
\text { soldat va exciter pour assaillir la } \\
\text { demeure. }\end{array}$ \\
\hline $\begin{array}{l}\text { Chrémès révèle à Thrason } \\
\text { que Pamphila est sa sœur. } \\
\text { Thrason renvoie ses } \\
\text { troupes. }\end{array}$ & $\begin{array}{l}\text { IV, } 7 \text { : Epitasis prioris partis in } \\
\text { fabula, est autem perridicula } \\
\text { Thrasone digna. }\end{array}$ & $\begin{array}{l}\text { IV, } 7: \text { Nœud [tension] de la première } \\
\text { intrigue de la pièce. Elle est tout à fait } \\
\text { ridicule, digne de Thrason. }\end{array}$ \\
\hline $\begin{array}{l}\text { Thaïs apprend de Pythias le } \\
\text { crime de Chéréa. }\end{array}$ & $\begin{array}{l}\mathrm{V}, 1: \text { Epitasis deuterioris partis } \\
\text { in fabula, deprehenso Chaerea. }\end{array}$ & $\begin{array}{l}\mathrm{V}, 1: \text { Nœud [tension] de la deuxième } \\
\text { intrigue de la pièce : Chéréa est pris. }\end{array}$ \\
\hline \multicolumn{3}{|l|}{ CATASTROPHE-DENOUEMENT } \\
\hline $\begin{array}{l}\text { Réconciliation de Thaïs } \\
\text { avec Chéréa, qui lui } \\
\text { demande son aide pour } \\
\text { épouser Pamphila. }\end{array}$ & $\begin{array}{l}\text { V, } 2 \text { [v. 875]: Placatur Thaïs, } \\
\text { estque parasceuè catastrophes. }\end{array}$ & $\begin{array}{l}\mathrm{V}, 2 \text { : Thaïs est apaisée, et c'est la } \\
\text { préparation du dénouement. }\end{array}$ \\
\hline $\begin{array}{l}\text { Pythias fait peur à } \\
\text { Parménon en lui annonçant } \\
\text { que Chéréa va être } \\
\text { terriblement châtié pour le } \\
\text { viol d'une citoyenne. }\end{array}$ & $\begin{array}{l}\mathrm{V}, 3: \text { Vt prolixior esset risus, } \\
\text { spargitur catastrophe in alias } \\
\text { personas, et terretur seruulus } \\
\text { Parmeno. }\end{array}$ & $\begin{array}{l}\mathrm{V}, 3 \text { : Pour augmenter les rires, le } \\
\text { dénouement «éclabousse » d'autres } \\
\text { personnages, et le jeune esclave } \\
\text { Parménon panique. }\end{array}$ \\
\hline $\begin{array}{l}\text { Thrason apprend que Thaïs } \\
\text { a choisi Phédria. }\end{array}$ & $\begin{array}{l}\mathrm{V}, 8: \text { Catastrophen orditur de } \\
\text { milite. }\end{array}$ & $\begin{array}{l}\mathrm{V}, \quad 8: \text { le poète commence le } \\
\text { dénouement de l'affaire du soldat. }\end{array}$ \\
\hline
\end{tabular}

Mélanchthon suit conjointement les deux intrigues dont les fils se succèdent avant de se mêler. La deuxième se greffe sur la première, quand Chéréa rencontre Parménon qui doit amener à Thaïs l'eunuque que lui offre Phédria (II, 3). Plus qu'une série de séquences, c'est un processus, un fil conducteur, que met en valeur Mélanchthon, processus long (deux actes pour la protase de la première intrigue) ou bref (le moment précis de la narration de la seconde intrigue en III, 5). La protase s'achève au moment où toutes les données sont connues par le spectateur qui doit alors se demander : que va-t-il arriver?

Il n'en va pas tout à fait de même pour l'épitase. Certes elle se prépare, comme le montre l'usage du terme parasceuè ; mais elle désigne ici, semble-t-il, le point culminant du péril. Ainsi, pour Thrason, lorsqu'il découvre qu'il ne peut récupérer Pamphila et que tous ses efforts ont été vains; ou pour Chéréa, lorsqu'il est fait prisonnier par Pythias et Thaïs. De même pour la catastrophe - qui se prépare (parasceuè) ou commence (orditur) - mais n'est effective que lorsqu'il y a mariage (Chéréa-Pamphila) ou accommodement (Thrason-Phédria ${ }^{26}$ ). À côté du terme parasceuè, on aura noté 
cependant celui d'occasio. Que désignent ces deux termes dans le repérage du dessein du Poète?

\subsection{L'occasion et la préparation}

D'origine rhétorique, ils semblent mettre en évidence le processus que constitue l'intrigue et dont les deux étapes essentielles sont, on l'a vu, l'épitase (moment culminant de la tension, du désordre) et la catastrophe (conversion, retour à l'ordre). Pour Cicéron (De Inv. I, XxvI, 38) l'occasio fait partie des circonstances de l'action avec le lieu, le temps, la manière et la possibilité. Il la définit ainsi : «occasio autem est pars temporis habens in se alicuius rei idoneam faciendi aut non faciendi opportunitatem" ( l'occasion est une période de temps qui offre les conditions favorables pour réaliser ou non une chose $\left.{ }^{27} »\right)$. L'occasion est donc une circonstance, c'est-à-dire un élément extérieur aux desseins des personnages. Si nous reprenons la note marginale à la scène 5 de l'acte IV de L'Andrienne, assez explicite: "Occasio catastrophes, nam senex casu interueniens, discutit periculum ", nous voyons que occasio entre en résonance avec casus, le hasard, qui correspond dans la pièce à l'arrivée de Criton, qui résout toutes choses. L' occasio désigne la circonstance extérieure, qui arrive - ou semble arriver - par hasard ; de même, dans L'Eunuque, l'arrivée soudaine de Chéréa.

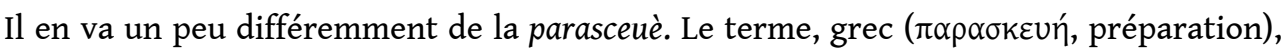
est employé par Denys d'Halicarnasse pour désigner les procédés qui rendent l'auditeur attentif, en particulier dans l'exorde ${ }^{28}$. Traduit chez Quintilien par praeparatio, il renvoie au dessein de l'orateur, à la vision globale qu'il a de son discours et de son but. Le meilleur plan du discours nécessite en effet de la part de ce dernier des choix réfléchis : faire ou non un exorde, choisir un exposé des faits qui remonte à l'origine ou plonge l'auditeur au milieu de l'événement, commencer par les preuves les plus fortes ou les plus faibles,

quand la Cause demande que l'on traitte certaines questions sans aucun préambule, quand au contraire ces questions ont besoin de préparation, quelles sont les choses que l'on peut dire tout d'un coup aux Juges, \& quelles sont celles où il faut les conduire comme pas à pas ${ }^{29}$.

Les deux termes, latin et grec, seront utilisés par Scaliger en un sens poétique, pour commenter la prudentia de Virgile. Au livre I de l'Énéide, si Énée se voit lui-même représenté au milieu des combats de Troie et trouve réconfort dans ces images, c'est une préfiguration - une préparation - du bon accueil que lui réservera Didon ${ }^{30}$.

La parasceuè n'intervient pas dans les notes de L'Andrienne mais dans celles de L'Eunuque . À quoi correspond-t-elle ? En III, 1, Gnathon conseille à Thrason d'éveiller la jalousie de Thaïs à l'égard de Pamphila pour contrer son rival Phédria. Ce conseil est, dit Mélanchthon, une préparation de l'épitase, c'est-à-dire de la montée du désordre que provoque la jalousie du soldat. En V, 2, l'indulgence de Thaïs à l'égard de Chéréa annonce la réconciliation finale que va sceller le mariage. Il s'agit donc bien d'étapes qui préparent le désordre ou le retour à l'ordre. Elles renvoient, non plus à des circonstances amenées de l'extérieur, mais à l'organisation interne de la pièce. Dans les deux cas cependant, il s'agit de l'agencement des éléments de l'intrigue, donc du dessein du poète, ou de l'art du dramaturge. 


\subsection{La mise en valeur de l'économie ou ductus de la pièce}

intéresse Mélanchthon, c'est donc de rendre compte du dessein du conduite de sa pièce. Le terme de conduite correspond au grec oikovouía, que Quintilien traduit par ductus et l'abbé Gedoyn par le français «suite ». Pour Quintilien, le terme concerne au premier chef le théâtre.

Il y a de plus une certaine suite qui répand un air de vraysemblance sur tout le sujet, \& qui fait que les choses paroissent amenées comme aux pieces Dramatiques. Car l'une doit naturellement conduire à l'autre; en sorte que la premiere bien racontée, l'auditeur devine ce qui va suivre ${ }^{31}$.

Là est bien la nouveauté pédagogique de Mélanchthon : permettre à ses élèves de suivre le fil - ou les fils - de l'intrigue et de comprendre l'art du poète à travers son dessein, dessein que reflète la cohérence de la pièce. Dramaturgie et rhétorique ici poursuivent le même but.

L'efficacité pédagogique de Mélanchthon se lit alors dans le parallèle qu'il établit implicitement entre la conduite des pièces de Térence et leur fonction éthique. Il faut revenir à la préface de L'Eunuque et aux lignes qui précèdent l'argument proprement dit :

Comoediae ut hominum uita, casus, consilia imitentur, pericula continent, quod et consiliis et fortunae, in periculis maxime locus est. Itaque cum discutere periculum ratione contendant personae, uaria finguntur consilia, in quibus alia fallunt, alia felicia sunt. Saepe plus casus potest quam ratio. Dominatur fere in rebus fortuna.

Les Comédies, en tant qu'elles imitent la vie, les hasards, les desseins des hommes, contiennent des périls, parce que les desseins comme la fortune interviennent surtout dans les périls. Donc, comme les personnages s'efforcent par la raison d'écarter le péril, ils imaginent de nombreux desseins qui échouent ou réussissent. Souvent le hasard peut plus que la raison. C'est la fortune qui domine presque toujours dans les affaires humaines.

Casus et fortuna d'un côté, consilia de l'autre : ce sont les deux principes qui régissent la vie humaine. Occasio d'un côté, parasceuè de l'autre: ce sont les deux principes qui régissent le ductus de la comédie. Ce parallèle reprend le lieu commun cicéronien de la comédie comme miroir de la vie, mais le praeceptor Germaniae va plus loin. L'opposition fortuna/consilia, d'ordre éthique, est devenue un mode de lecture des pièces, donc un outil critique, voire poétique, pour de futurs dramaturges.

La lecture de Térence par Mélanchthon semble donc un jalon d'importance dans l'histoire de l'analyse des comédies tout autant que dans celle de l'enseignement. À l'origine de l'analyse, le projet pédagogique use des outils rhétoriques pour reconsidérer la conduite d'une comédie à la manière de celle d'un discours. Ce faisant, Mélanchthon enseigne assurément déjà la pratique oratoire à ses élèves, mais il met surtout en valeur l'art du dramaturge, jetant par là même les bases d'une poétique de la comédie, que reprendront en partie Peletier du Mans et Scaliger. Sa méthode, dont on a tenté ici de présenter une description, tient à l'usage critique et poétique qu'il fait de la rhétorique, usage associé à la lecture dramaturgique de la comédie comme processus organisé en trois mouvements tissés ensemble, la protase, l'épitase et la catastrophe, selon le modèle transmis par Évanthius. Processus organisé, qui ne tient pas compte des actes ni des scènes, et propose par conséquent une lecture " organique » des pièces en insistant sur la façon dont les parties sont habilement disposées et liées entre elles. Pour laisser le dernier mot à Quintilien, corpus [est], non membra ${ }^{32}$. 


\section{NOTES}

1. Voir K. Meerhoff, Entre logique et littérature. Autour de Philippe Mélanchthon, Orléans, Paradigme, 2003, chap. I, p. 12. J'utilise ici l'édition de 1528 (Cologne, Eucharius Cervicornus, disponible en ligne sur books.google.fr) qui comporte les notes marginales de Mélanchthon (précédées d'une astérisque) et celles de Paulus Malleolus.

2. Voir l'épître « Aux professeurs» (« Paedagogis »), non paginée, qui ouvre l'édition de 1528 ainsi que l'« Adhortatio Philippi Melanchthonis de legendis Tragoediis et Comoediis » (1545) non paginée, Terentii comoediae sex [...], Francfort, Ioan. Eichorn, 1560 : « Est enim modis omnibus efficiendum, ut mirentur, ut ament hunc authorem pueri. Id ita fiet, si rectissime intellexerint. » (« Il faut en effet faire en sorte par tous les moyens que les enfants admirent, qu'ils aiment cet auteur. Ce sera le cas s'ils l'ont compris à la perfection »).

3. "Adhortatio ", op. cit. Le but de la comédie est en ce sens proche de celui de la tragédie qui veut « inciter les âmes rudes et violentes à se modérer et à refréner leurs désirs excessifs » («ut rudes ac feros animos [...] flecterent ad moderationem, et frenandas cupiditates »). La différence est une différence de degré : «les exemples de morale et d'éloquence sont plus illustres dans les Tragédies que dans les Comédies » (« et morum et eloquentiae exempla sunt illustriora in Tragoediis, quam in Comoediis »).

4. K. Meerhoff, op. cit., p. 17-19. Dans ses Elementa rhetorices de 1532, Mélanchthon propose, aux côtés des genres délibératif, judiciaire et démonstratif, un quatrième genre qu'il appelle didascalique. Ce genre s'appuie sur la Dialectique et vise à enseigner (docere).

5. "Nam et fabulae ad actum scaenarum compositae argumenta dicuntur. [...] Quo apparet omnem ad scribendum destinatam materiam ita appellari. ", Institution oratoire, V, 10, 9-10 (la traduction est celle de l'abbé Gedoyn, Paris, Grégoire Dupuis, 1718).

6. Cicéron, De l'Invention, I, XIX (27), Paris, Les Belles Lettres, 1994.

7. Les periochae ou argumenta en vers des comédies de Térence, dus à Sulpice Apollinaire de Carthage, ont été composés au II $^{\mathrm{e}}$ siècle. On les retrouve, sauf erreur, dans toutes les éditions.

8. Lyon, Mathias Bonhomme, 1560. Le terme d'occasio semble désigner ici la situation dramatique. 9. " Exigendum ab ipsis pueris, ut suo Marte argumenta fabularum condant, ut locos aliquot retexant, et pluribus exponant, et tanquam ceram refingant. Quae exercitatio praeterquam quod familiariorem hunc Poetam facit, facundiam etiam alit " (préface à L'Andrienne, qui suit l'argument, éd. citée, non paginée). On sait que cette pratique deviendra un des piliers de la rhétorique scolaire jusqu'à la fin du XIX ${ }^{\mathrm{e}}$ siècle.

10. Ibid.

11. Art poétique, v. 146-152. C'est en s'inspirant de ce passage que Jacques Amyot, dans sa préface à la traduction des Éthiopiques d'Héliodore (Paris, 1548), distingue entre ordre naturel et ordre artificiel.

12. « ubi ab initiis incipiendum, ubi more Homerico a mediis uel ultimis » (Institution oratoire, VII, 10, 11).

13. Aelii Donati in Andriam Terenti commentum, Hyperdonat - Collection d'éditions numériques de commentaires anciens avec traduction, commentaire et annotation critique, disponible sur http://hyperdonat.tge-adonis.fr (pour le texte et la traduction). Les arguments en vers de Sulpice Apollinaire, bien que plus concis, commencent aussi par donner des éléments extérieurs et antérieurs à l'intrigue avant de la raconter.

14. Ibid. : "Perspecto argumento scire debemus hanc esse uirtutem poeticam, ut a nouissimis argumenti rebus incipiens initium fabulae et originem narratiue reddat spectatoribus auctoremque praesentem scilicet ibi exhibeat, ubi finis est fabulae. hunc enim orbem et circulum poeticae uirtutis non modo secuti sunt tragici comicique auctores, sed Homerus etiam et Vergilius tenuerunt. » 
15. Terentius, a M. Antonio Mureto locis prope innumerabilibus emendatus. Eiusdem Mureti argumenta in singulas comoedias, et annotationes [...], Venise, Paul Manuce, 1555.

16. Cette division, communément traduite à l'âge classique par exposition, nœud, dénouement, mais inconnue d'Aristote, vient sans doute des Alexandrins et a été transmise au monde latin par Donat et, avant lui, par Évanthius (De fabula, 4, 5, disponible sur http://hyperdonat.tge-adonis.fr). Voir sur ce point $\mathrm{C}$. Nicolas, « Fins d'actes et fins de scènes dans les comédies de Térence lues par Donat ", Commencer et finir. Débuts et fins dans les littératures grecque, latine et néolatine, textes réunis par B. Bureau et C. Nicolas, Université Jean Moulin, Edition CERGR, 2008 (2 vols), vol. 2, p. 613. Je reviendrai plus loin sur la traduction de ces termes.

17. « Préface à L'Andrienne », op. cit., non paginé : «Et tota fabula similis est orationi generis suasorii ».

18. Héros et orateurs. Rhétorique et dramaturgie cornéliennes, Droz, 1990, p. 271.

19. Mélanchthon, Elementa rhetorices [1532], éd. et trad. allemande de V. Wels, Berlin, Weidler Buchverlag, 2001, p. 70.

20. Ces définitions sont vouées à être reprises. Chez Scaliger (Poetices libri septem [1561], I, 9, éd. L. Deitz et G. Vogt-Spira, Stuttgart-Bad Cannstatt, Frommann-Holzboog, 1994-2011, vol. 1, p. 152), la protase est ainsi définie comme « la partie où est exposé et narré l'essentiel de la situation sans révélation de la fin " ("Protasis est, in qua proponitur et narratur summa rei sine declaratione exitus »). Peletier du Mans parle, lui, de «proposition du fait »: «La Comédie a trois parties principales sans le Prologue. La première, est la proposition du fait, au premier Acte : laquelle est appelée des Grecs Protasie. Et en elle s'explique une partie de tout l'Argument, pour tenir le Peuple en attente de connaître le surplus. La seconde, est l'avancement ou progrès, que les Grecs disent Épitasie. C'est quand les affaires tombent en difficulté, et entre peur et espérance. La tierce, est la Catastrophe, soudaine conversion des choses au mieux. Dont je ne parlerai plus au long. Car les Comédies de Térence sont entre les mains de chacun » (Art Poétique, II, 7, éd. Francis Goyet, Paris, Le Livre de poche, 1990, p. 302). Sur ces notions, voir aussi C. Ailloud-Nicolas, Le dénouement dans les pièces en un acte de Marivaux: structure dramaturgique, sous la direction de Ch. Hamon, Université Lyon II, 2003, disponible en ligne sur : theses.fr, première partie.

21. Sur le sens rhétorique de la terminologie mélanchthonienne, voir aussi T.W.Baldwin, Shakspere's five act structure: Shakspere's Early Plays on the Background of Renaissance Theories of FiveAct Structure From 1470, Urbana, University of Illinois Press, 1947, chap. VIII, p. 177-178. Mais Baldwin reproche à Mélanchthon de ne pas tenir compte de la division en actes sans voir, à mon sens, l'originalité de la lecture du réformateur.

22. Il n'est pas exclu que Mélanchthon s'inspire aussi de la distinction désis/lusis qu'Aristote développe au chapitre 18 de sa Poétique, ouvrage qu'il cite explicitement dans sa préface à L'Andrienne.

23. C'est-à-dire avant la fin de la pièce (où l'on relevait le rideau, baissé pour montrer le décor). Le texte et la traduction, comme cette précision, proviennent du site Hyperdonat, déjà cité.

24. Cette translatio rhetorica correspond, semble-t-il, à la translatio criminis (transfert du crime sur autrui) qui est, dans un contexte judiciaire, une des trois manières de se défendre d'une accusation fondée, avec la purgatio (circonstances atténuantes) et la deprecatio (demande de clémence). Le verbe imputari appartient au vocabulaire juridique. Mélanchthon décrit ces trois manières dans ses Elementa rhetorices [1532], op. cit., p. 96.

25. Voir dans ce même numéro la rubrique « Atelier ». On pourrait traduire ici : «c'est le moment où la tension commence à croître » ou « c'est le moment où le péril se fait sentir ».

26. Christian Nicolas notait déjà cette indécision à propos de Donat. Mais Donat est très loin de repérer le processus aussi nettement que Mélanchthon.

27. De Inventione, I, XXVII [40].

28. Ars rhetorica, X, 13, éd. I. Reiske, Leipzig, Weidmann, 1775, p. 388.

29. "qua in causa proponendae prohoemiis quaestiones, qua praeparatione praemuniendae, quid iudicis animus accipere possit statim dictum, quo paulatim deducendus " (Institution oratoire, VII, 10, 12, trad. 
Gedoyn). Les praeparationes étaient déjà évoquées à propos de la narratio (op. cit., IV, 2, 55 et 57 où Quintilien précise que la meilleure préparation est celle qui ne se voit pas: «Optimae uero praeparationes erunt quae latuerint »).

30. Énéide, I, v. 459-493. Scaliger note : "Similis praeparatio in primo. Cum enim recipiendus esset Aeneas, proponit hoc intellegendum ex pictura ; in ea namque ipse quoque pictus erat » (Poetices libri septem, op. cit., III, 25, « Prudentia », p. 326). L'abbé d'Aubignac cite ce passage dans La Pratique du théâtre, II, VIII, « De la Préparation des Incidents », éd. H. Baby, Paris, Champion, 2001, p. 194.

31. "Est autem quidam et ductus rei credibilis, qualis in comoediis etiam et in mimis. Aliqua enim naturaliter secuntur et cohaerent, ut, si bene priora narraueris, iudex ipse quod postea sis narraturus expectet » (op. cit., IV , 2, 53, trad. Gedoyn). La notion de ductus se trouve aussi chez Donat, lorsqu'il utilise par exemple le verbe ducere dans son argument de L'Andrienne: " totus error inenodabilis usque ad eum finem est ductus " (cité au début de cet article). Pierre Davantès, à propos des argumenta, utilisait le terme grec translittéré, œconomia. Sur la notion de ductus, voir aussi M. Carruthers, Machina memorialis. Méditation, rhétorique et fabrication des images au Moyen Âge, traduit de l'anglais par F. Durand-Bogaert, Paris, Gallimard, « Bibliothèque des histoires », 2002, p. 327 sq.

32. Quintilien, op. cit., VII, 10, 16, à propos de la dispositio des parties du discours : «Corpus sit, non membra » («Qu'elles forment un corps, et non des membres »).

\section{AUTEUR}

\section{CHRISTIANE DELOINCE-LOUETTE}

Université Grenoble-Alpes - UMR 5316 (Litt\&Arts/RARE) 